\title{
Force Sensing Robot Fingers using Embedded Fiber Bragg Grating Sensors and Shape Deposition Manufacturing
}

\author{
Yong-Lae Park ${ }^{1}$, Kelvin Chau ${ }^{2}$, Richard J. Black ${ }^{2}$ and Mark R. Cutkosky ${ }^{1}$ \\ ${ }^{1}$ Center for Design Research, Stanford University \\ Stanford, CA 94305-2232, USA \\ contact: ylpark@stanford.edu \\ ${ }^{2}$ Intelligent Fiber Optic Systems Corporation (www.ifos.com) \\ 2363 Calle Del Mundo, Santa Clara, CA 95054, USA \\ contact: info@ifos.com
}

\begin{abstract}
Force sensing is an essential requirement for dexterous robot manipulation. Although strain gages have been widely used, a new sensing approach is desirable for applications that require greater robustness, design flexibility and immunity to electromagnetic noise. An exoskeletal force sensing robot finger was developed by embedding Fiber Bragg Grating (FBG) sensors into a polymer-based structure. Multiple FBG sensors were embedded into the structure to allow the manipulator to sense and measure both contact forces and grasping forces. In order to fabricate a three-dimensional structure, a new shape deposition manufacturing (SDM) process was explored. The sensorized SDM-fabricated finger was then characterized using an FBG interrogator. A force localization scheme is also described.
\end{abstract}

\section{INTRODUCTION}

Future robots are expected to free human operators from difficult and dangerous tasks requiring high dexterity in various environments. One example is an extra-vehicular repair of a manned spacecraft that would otherwise require hazardous work by human astronauts. Another example is robotic surgery in which accurate manipulation is crucial. Operating complicated tools and performing delicate tasks require a manipulator of great precision and coordination. Therefore, force sensing is one of the most critical requirements for this type of robot control.

However, compared to even the simplest of animals, today's robots are impoverished in terms of their sensing abilities. For example, a single spider can contain as many as 325 mechanoreceptors on its legs [2], in addition to hair sensors, chemical sensors, etc. [1], [26]. Mechanoreceptors such as the slit sensilla of spiders [2], [11] and campaniform sensilla of insects [20], [27] are especially concentrated near the joints, where they provide the animals with extensive information about loads imposed on the limbs - whether due to normal activities such as running or grasping prey, or as the result of unexpected events such as collisions. In contrast, robots generally have a modest number of mechanical sensors, often associated with actuators or concentrated in a special device such as a force sensing wrist. As a result,

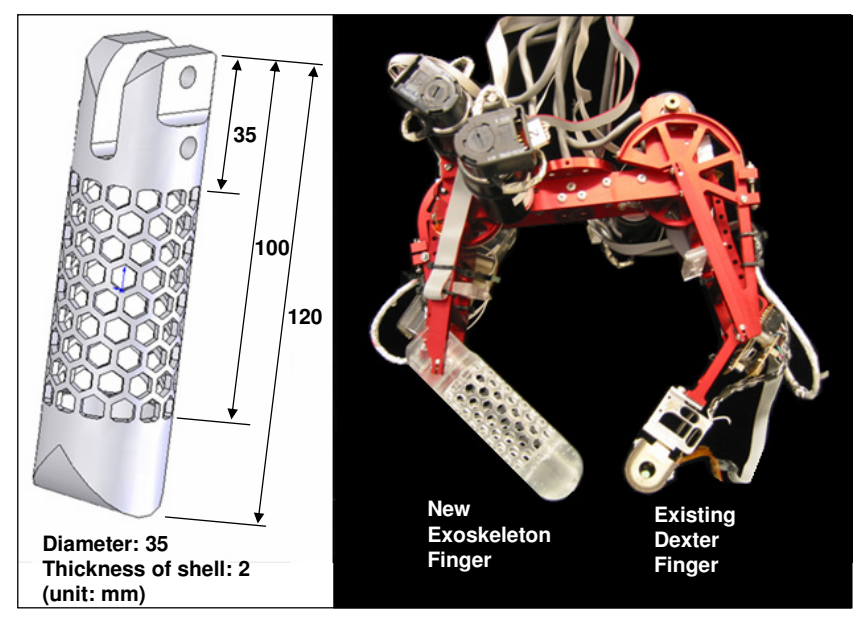

Fig. 1. Prototype dimensions and installation on Dexter manipulator

robots often poorly identify and respond to unexpected and arbitrarily-located impacts.

The work in this paper is part of a broader effort aimed at creating light-weight, rugged appendages for robots that, like the exoskeleton of an insect, feature embedded sensors so that the robot can be more aware of both anticipated and unanticipated loads in real time. We focus on a particular class of optical sensors, Fiber Bragg Grating (FBG) sensors, which have been identified as promising for space robotics and other applications where high sensitivity, multiplexing capability, immunity to electromagnetic noise, small size and resistance to harsh environments are particularly desirable. In addition, we note the biosafe and inert nature of optical fibers making them attractive for medical robotics.

FBGs reflect light with a peak wavelength that shifts in proportion to the strain to which they are subjected. This wavelength shift provides the basis for strain sensing with typical values for the sensitivity to an axial strain being approximately $1.2 \mathrm{pm} /$ microstrain at $1550 \mathrm{~nm}$ center wavelength [6]. With the appropriate FBG interrogator, submicrostrain resolution measurements are possible. In addition, the strain response is linear with no indication of 

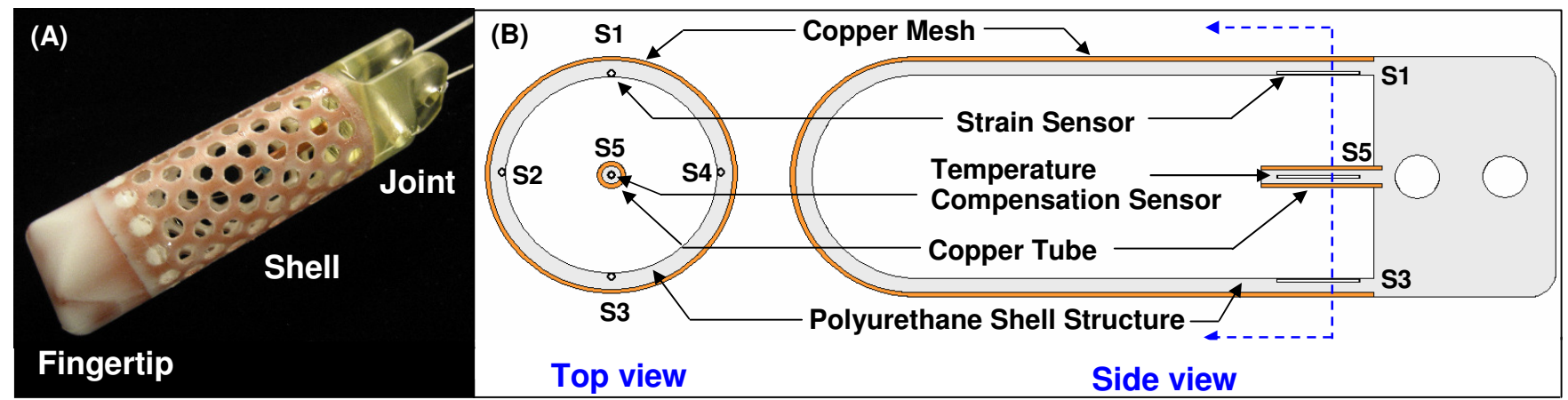

Fig. 2. (A) Complete finger prototype (B) Cross-sectional views (s1-s4: strain sensors, s5: temperature compensation sensor)

hysteresis at temperatures as high as $370^{\circ} \mathrm{C}$ [21] and, with appropriate processing, to over $650^{\circ} \mathrm{C}$ [5], [23]. Multiple FBG sensors can be placed along a single fiber and optically multiplexed.

FBG sensors have previously been surface attached to or embedded in metal parts [6], [18] and composites [29] to monitor stresses. To our knowledge this is their first application in hollow, multi-material structures made by a rapid-prototyping process. In this paper we describe the rapid prototyping process and its adaptation to allow the embedding of optical fiber sensors. The results of a first prototype revealed the need to embed a thermal shield to reduce the effects of external temperature variations. Accordingly, a second prototype was developed with an embedded copper mesh in addition to four fiber optic sensors. The results of tests performed on this prototype are reported and the implications for use in force control as well as collision detection are discussed. We then discuss the enabling optical interrogation technology for reading the strains to which the FBGs are subjected [10], [12], [13], [22]. We conclude with a discussion of future work, including extensions to a larger number of sensors so that a more complete picture of external force magnitudes and contact locations can be obtained.

\section{DESIGN CONCEPT}

Since the prototype will replace the lower finger of the Dexter [16] manipulator as shown in Figure 1, its dimensions have been set accordingly. Figure 2 shows the completed finger prototype and its cross-sectional views. The finger can be divided into three parts: fingertip, shell, and joint. The fingertip and shell are hollow, exoskeletal structures. Four FBG sensors were embedded into the shell for strain measurement, and one FBG sensor was placed in the middle of the structure for temperature compensation.

\section{A. Exoskeletal Structure}

The exoskeletal structure is light weight while maintaining relatively high strength. Since the structure deforms not only locally but globally depending on the location of force application, the finger is able to measure and localize applied forces. This is useful for both grasp force measurement and collision detection.
A plastic shell fingertip has been proposed by Voyles et al. [30] for extrinsic tactile sensing using electrorheological fluids. However, it was necessary to make an additional cantilever beam structure to install strain gages to obtain force-torque information for intrinsic tactile sensing [4]. The prototype discussed in this paper requires only a shell structure to provide force information for both extrinsic and intrinsic sensing.

\section{B. Hexagonal Shell Pattern}

The prototype has a hexagonally patterned shell. This pattern allows the structure to concentrate stresses and strains on the narrow ribs, facilitates embedded sensor placement and has an added effect of amplifying the sensor signal.

Although two other regular polygons, triangles and squares, can also be used exclusively to form the shell pattern, the hexagon minimizes the ratio of perimeter to area [24], as proved by Hales [17]. In addition, the hexagonal cells avoid sharp interior corners which could reduce the fatigue life. In summary, the hexagonal structure can minimize the amount of material for fabrication and the weight of the part while providing high structural strength.

\section{Creep Prevention and Thermal Shielding}

Polymer structures unavoidably experience greater creep than metal structures. Creep adversely affects the linearity and repeatability of the embedded sensor output, both of which are mainly dependent on the stiffness and resilience of the structure. In addition, thermal changes can affect the FBG strain sensor outputs. Drawing inspiration from [15], a copper mesh (080X080C0055W36T, TWP Inc., Berkeley, California, USA) was embedded into the outside of the shell, to reduce creep and provide thermal shielding. The high conductivity of copper expedites distribution of heat applied from outside the shell and creates a more uniform temperature gradient inside the shell.

\section{Strain Sensor Configuration}

More sensors provide more information and make the system more reliable. However, since more sensors require more time and/or processing capacity to handle the larger amount 


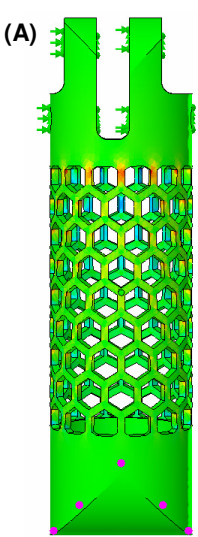

Front View

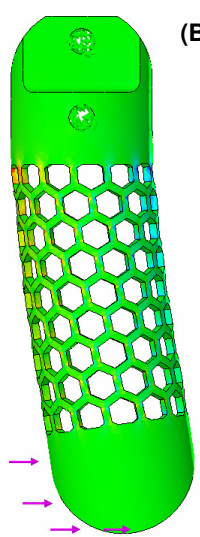

Right View
(B)

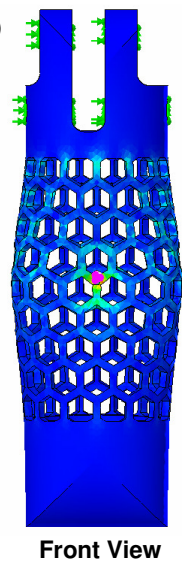

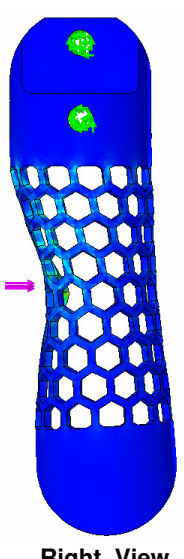

Right View
Fig. 3. Finite element analysis of finger prototype

of data, the optimal sensor design should be considered as discussed by Bicchi [3]. Ultimately, the force information we would like to obtain from our system includes longitudinal location, latitudinal location, magnitude of applied force, and orientation of the force vector. For the present, we assume forces are applied only in a normal direction to the surface to simplify the system. Since this assumption reduces the number of unknowns to three, a minimum of three linearly independent sensors are needed. In the prototype, four strain sensors were embedded in the shell.

Before starting fabrication, finite element analysis was conducted to determine the sensor locations. Figure 3 shows strain distributions when different types of forces are applied to the shell and to the fingertip. Strain is most concentrated at the top of the shell where it is connected to the joint. Therefore, four sensors were embedded at $90^{\circ}$ intervals into the first rib of the shell, closest to the joint, as shown in Figure 2.

\section{E. Temperature Compensation}

Since embedded FBG sensors are sensitive to temperature change as well as strain change, it is necessary to isolate thermal effects from mechanical strains. Various complicated temperature compensation methods have been proposed, such as use of dual-wavelength superimposed FBG sensors [9], [32], saturated chirped FBG sensors [33], and an FBG sensor rosette [19]. In contrast, a simpler method is to use an isolated, strain-free FBG sensor to directly measure the thermal effects. Subtracting the wavelength shift of this temperature-compensation sensor from that of any other sensor corrects for the thermal effects on the latter [25]. An important assumption in this method is that all sensors are at the same temperature. Our prototype has one temperature compensation sensor in the hollow area in the middle of the shell as shown in Figure 2. Although the temperature compensation sensor is physically removed from the strain sensors, the copper heat shield is expected to create a more uniform temperature gradient inside the shell. The temperature compensation sensor was encapsulated in a stiff copper
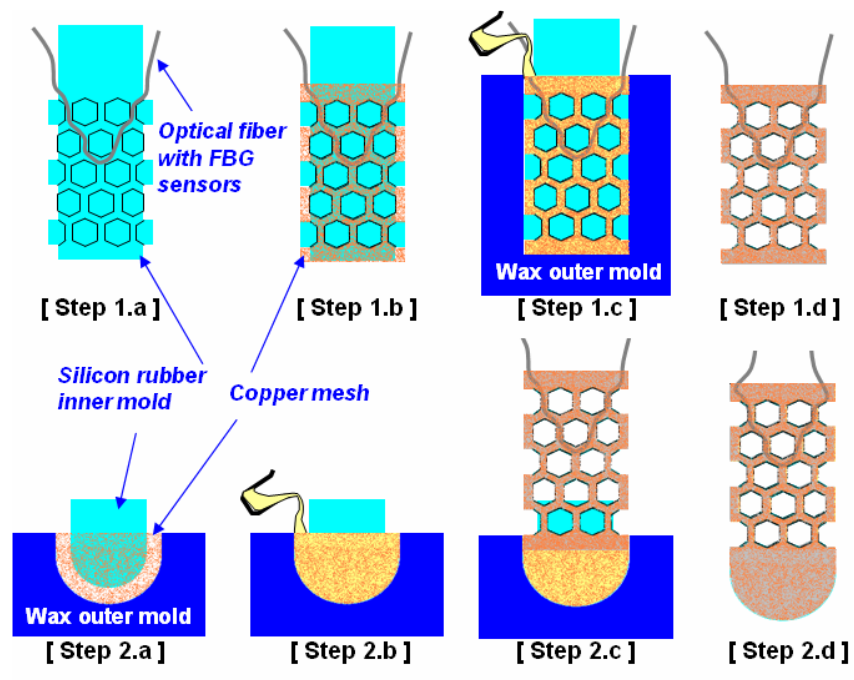

[ Step 1.d]

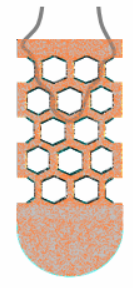

[ Step 2.d]
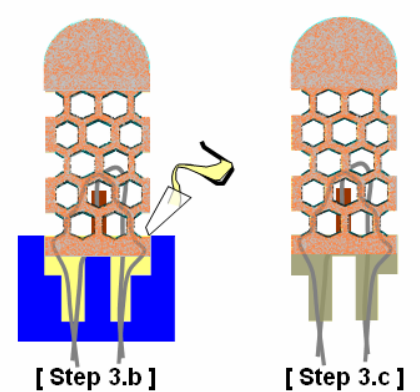

Fig. 4. Modified SDM process for prototype fabrication: [Step 1] Shell part fabrication (a) Prepare a silicone rubber inner mold and place optical fibers with FBG sensors (b) Wrap the inner mold with copper mesh (c) Enclose the inner mold and copper mesh with a wax outer mold and pour liquid polyurethane (d) Remove the inner and outer molds when the polyurethane cures, [Step 2] Fingertip part fabrication (a) Prepare inner and outer molds and place copper mesh (b) Pour liquid polyurethane (c) Place the cured shell part into the uncured polyurethane (d) Remove the molds when the polyurethane cures, [Step 3] Joint part fabrication (a) Prepare an outer mold and place a temperature compensation sensor structure (b) Place the cured shell and fingertip part into the uncured polyurethane (c) Remove the outer mold when the polyurethane cures

tube attached at only one end to the joint, and therefore is expected to experience no mechanical strain, regardless of external forces applied to the finger.

\section{SDM FABRICATION PROCEDURE}

Figure 4 shows the steps of the Shape Deposition Manufacturing (SDM) process [31] for the finger prototype fabrication. It is difficult to make hollow three-dimensional parts using conventional SDM processes, since only the top of the part is accessible for machining. Therefore, a modified SDM process was developed and applied for the fabrication of the finger. The prototype was cast in steps with no direct machining. The first step is molding of the shell. The outer mold is made of hard wax to maintain the overall shape. In contrast, the inner mold is hollow and made of soft silicone rubber, which can be manually deformed and removed when the polyurethane is cured. The strain sensors and copper mesh are embedded in this step. The second step is fingertip casting, which uses a separate mold and occurs after the shell is fully cured. As it cures, the polyurethane for the fingertip 


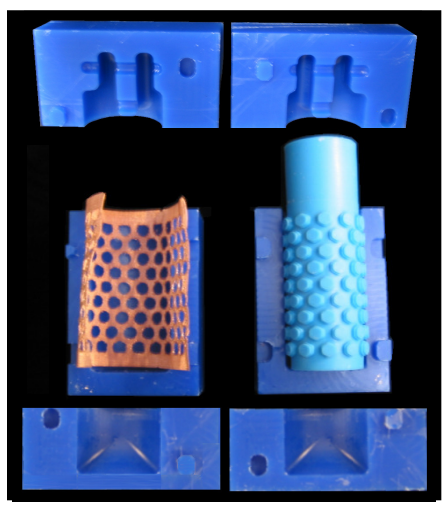

Fig. 5. Molds and copper mesh used in modified SDM fabrication process

bonds to the cured polyurethane of the shell. In the final step, the joint is cast. As with the fingertip, the joint bonds to the cured shell. Since the joint is not hollow, an inner mold is not needed during this step. Since the joint has no copper mesh, it was cast using a hard polyurethane (Task 9, SmoothOn, Easton, Pennsylvania, USA) to reduce creep, while the shell and fingertip were both cast from softer polyurethane (Task 3, Smooth-On, Easton, Pennsylvania, USA). The actual molds and copper mesh used in this modified SDM process are shown in Figure 5.

\section{Force Sensing Test and Evaluation}

Three different sets of tests were carried out to evaluate the static, dynamic, and thermal performance of the prototype. The static tests show how linear and repeatable the system is, the dynamic tests show how responsive the system is, and the thermal tests show how well the system compensates for errors caused by temperature change.

\section{A. Static Tests}

Static forces were applied to two different locations on the finger: shell and fingertip. Figures 6 and 7 show the force locations and the responses of two of the four sensors. Applying a force to the shell yielded sensitivities of 0.024 $\mathrm{nm} / \mathrm{N}$ and $-0.0044 \mathrm{~nm} / \mathrm{N}$ for sensor $\mathrm{A}$ and $\mathrm{B}$, respectively. The optical system can resolve wavelength changes of $0.5 \mathrm{pm}$ or less, corresponding to $0.015 \mathrm{~N}$ or less for the minimum detectable force change. Note that the A sensor, being on the same side of the shell as the contact force, has a much higher sensitivity to it. Applying a force to the fingertip yielded sensitivities of $0.032 \mathrm{~nm} / \mathrm{N}$ and $-0.029 \mathrm{~nm} / \mathrm{N}$. In this case, the location of the force results in roughly equal strains at both sensors. For a given location, the ratio of the two sensor outputs is independent of the magnitude of the applied force. The effect of location is discussed in Section V (Contact Force Localization). The system shows a maximum of $5.3 \%$ and $3.9 \%$ deviations from linear responses for shell and fingertip tests, respectively.

\section{B. Dynamic Tests}

Dynamic force tests were conducted by subjecting the system to a step input, which was generated by quickly
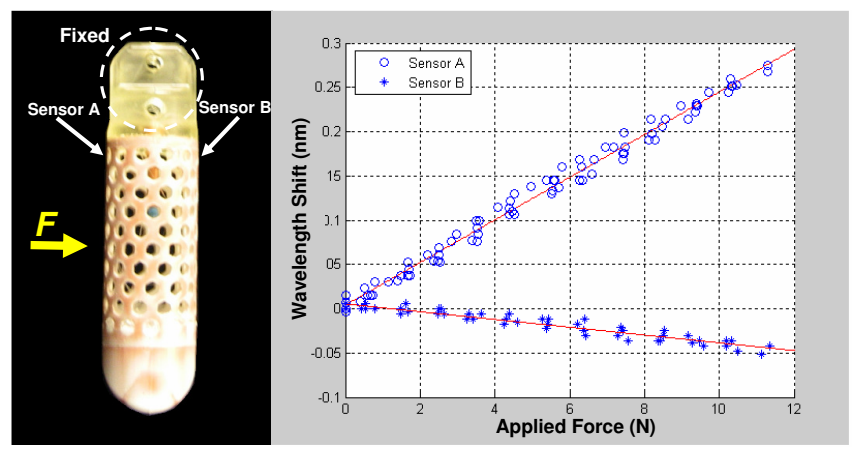

Fig. 6. Shell contact force response test
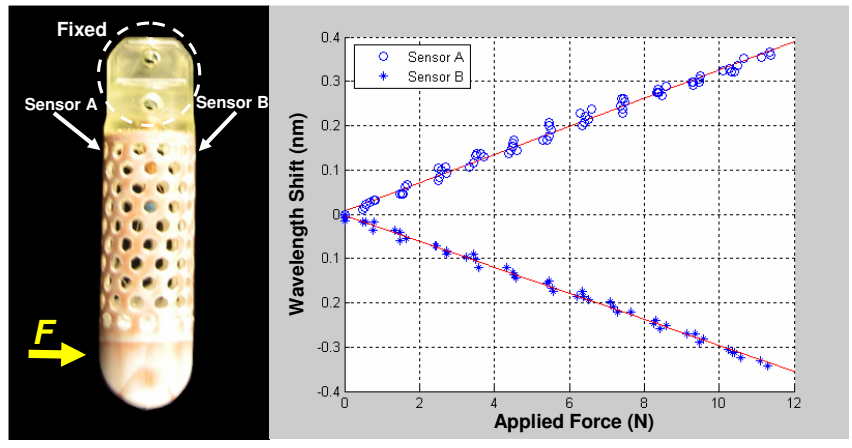

Fig. 7. Fingertip force response test

removing a weight of $200 \mathrm{~g}(1.962 \mathrm{~N})$. The sensor was sampled at $10 \mathrm{kHz}$, and the output is shown in Figure 8. Rise and settling times were approximately 0.01 second and 0.05 seconds, respectively, and less than $15 \%$ overshoot was observed. Since the linear signal to noise ratio is 25.25 , this structure can measure dynamic forces as small as $0.01 \mathrm{~N}$ at rates of approximately $20 \mathrm{~Hz}$.

\section{Thermal Effect Tests}

Figure 9 shows a typical thermal test result. Over a three minute period, the fingertip was loaded and unloaded while the temperature was decreased from $28.3^{\circ} \mathrm{C}$ to $25.7^{\circ} \mathrm{C}$. The ideal (temperature invariant) sensor output is indicated by the dashed line. Experiment results show that use of the

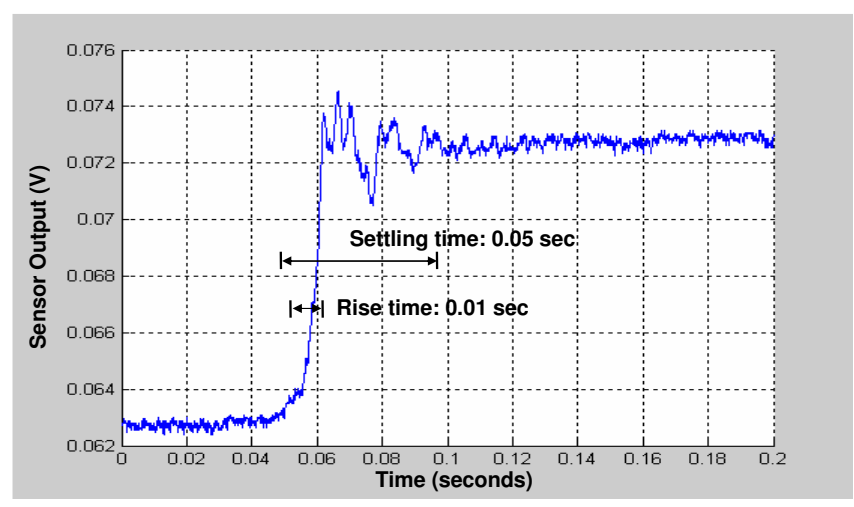

Fig. 8. Step response of strain sensor 


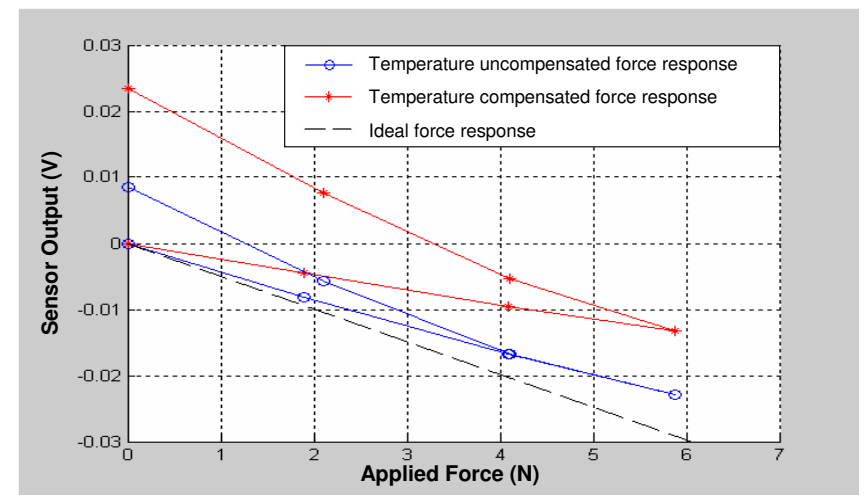

Fig. 9. Varying temperature test result showing partial temperature compensation provided by the central sensor

temperature compensation sensor reduces thermal effects somewhat. However, a more accurate compensation design is desired in the next prototype.

\section{Contact Force Localization}

\section{A. Longitudinal Location}

Longitudinal localization requires some understanding of structural deformation of the shell. Figure 10 shows simplified two-dimensional diagrams of the prototype. When a force is exerted at a certain location, as shown in (A), the structure will deform and sensors $A$ and $B$ will measure strains $\varepsilon_{A}$ and $\varepsilon_{B}$, respectively as indicated. This situation can be decomposed into two separate effects, as shown in (B) and (C). By superposition, $\varepsilon_{A}=\varepsilon_{1}+\varepsilon_{2}$ and $\varepsilon_{B}=\varepsilon_{3}$. Therefore, if the ratio of $\varepsilon_{A}$ to $\varepsilon_{B}$ is known, we can estimate $d$, the longitudinal location of the force. Figure 11 shows the plot of experimental ratios of $\varepsilon_{A}$ to $\varepsilon_{B}$ as a function of $d$.

There is some ambiguity in the localization, since two values of $d$ result in the same ratio. However, if we let $d_{0}$ be the distance at which $\varepsilon_{A} / \varepsilon_{B}$ is minimized, and we restrict ourselves the region $d>d_{0}$, we can resolve the ambiguity. Further, if we modify the manufacturing process to place the sensors closer to the other surface of the shell, $d_{0}$ approaches 0 and we can localize an applied force closer to the joint.

\section{B. Latitudinal Location}

Latitudinal location can be approximated using centroid and peak detection as discussed by Son et al. [28]. Only one point contact force is assumed in this method. Figure 12 (A) shows a cross sectional view of the finger with four strain sensors and an applied contact force indicated, and figure 12 (B) shows its corresponding sensor signal outputs. The two sensors closest to the force location will experience positive strains (positive sensor output), and the other two sensors negative strains (negative sensor output), regardless of the longitudinal location of the force, if $d>d_{0}$. However, since all the sensor signals must be non-negative to use the centroid method, all signal values must have the minimum signal value subtracted from them. Then, we can find the

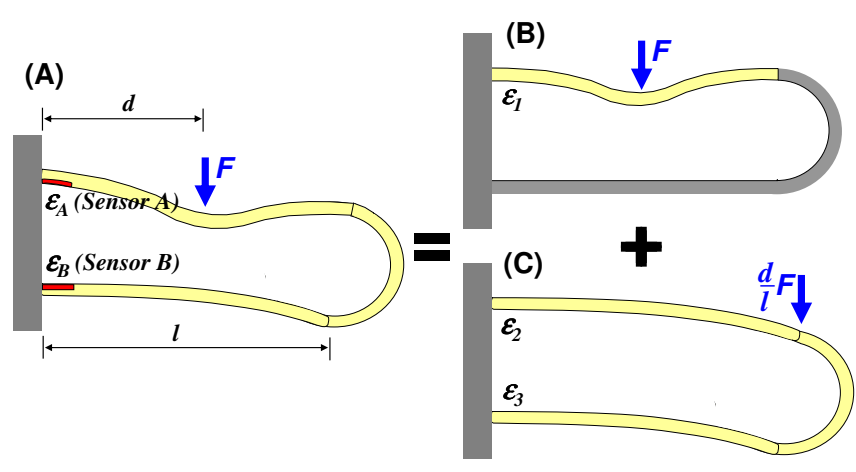

Fig. 10. 2D simplified shell structure and deformations

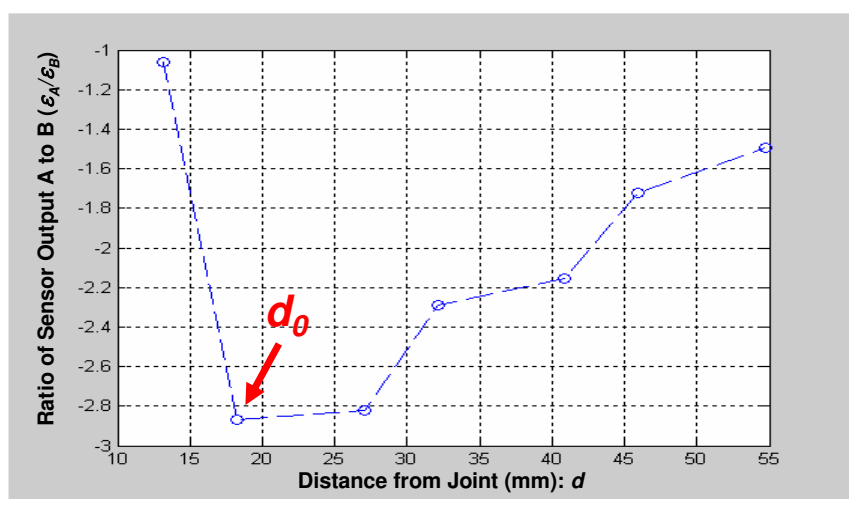

Fig. 11. Strain ratio of sensor A to $\mathrm{B}\left(\varepsilon_{A} / \varepsilon_{B}\right)$ for several locations of force application along the length of the finger

angular orientation $\theta$ of the contact force:

$$
\theta=\frac{\sum \phi_{i} S_{i}^{\prime}}{\sum S_{i}^{\prime}}-\alpha
$$

for $i=1,2,3,4$, where $S_{i}^{\prime}=S_{i}-\min \left\{S_{1}, S_{2}, S_{3}, S_{4}\right\}$, $\phi_{1}=\alpha$ and $\phi_{k}=\phi_{k-1}+\frac{\pi}{2}$, for $k=2,3,4$ (if $\phi_{k} \geq 2 \pi$, $\left.\phi_{k}=\phi_{k}-2 \pi\right), S_{i}$ is the output signal from sensor $i$, and $\alpha$ is the clockwise angle between sensor 1 and the sensor with the minimum output signal value.

This method produced errors less than $2^{\circ}$, corresponding to less than $0.5 \mathrm{~mm}$ on the perimeter, and an offset of $1.5^{\circ}$ in the FEM simulation. However, experimental data gave an offset of approximately $5^{\circ}$, likely due to manufacturing tolerances in the placement of the sensors.
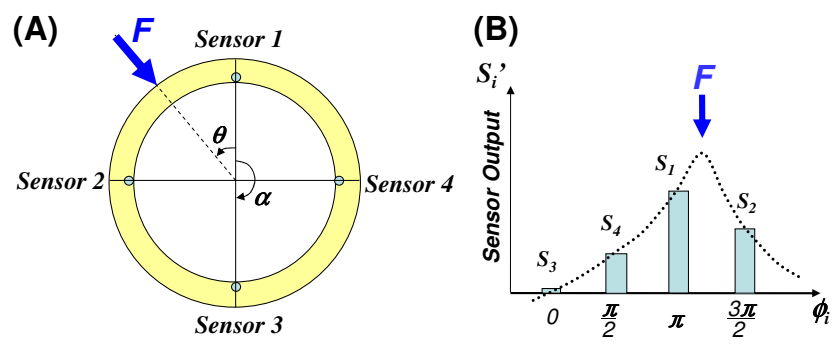

Fig. 12. (A) Top view of the prototype showing embedded sensors and force application (B) Plot of sensor signal outputs 


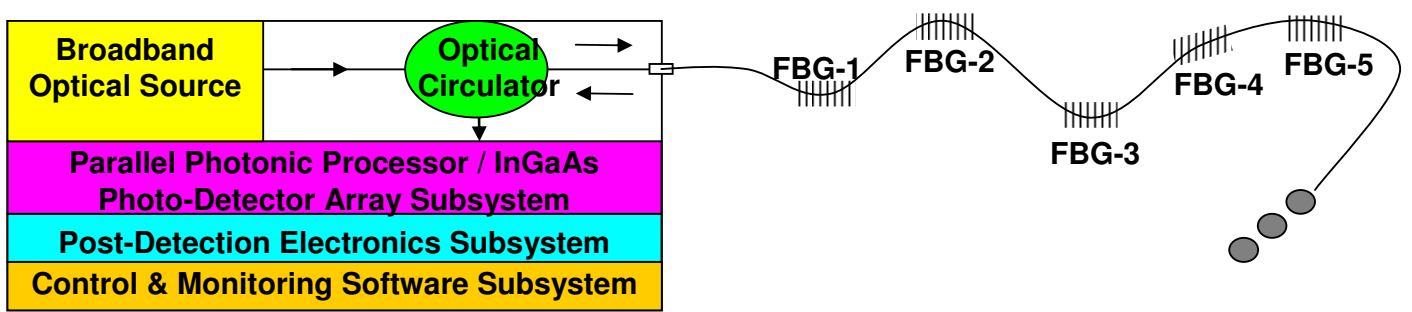

Fig. 13. Functional diagram (not-to-scale) of FBG interrogator based on a photonic parallel spectral processor which simultaneously processes signals reflected from all Fiber Bragg Gratings (FBGs).

\section{IFOS FBG SENSOR INTERROGATOR}

In the tests for this paper, the FBG sensors were interrogated by a new version of the I-Sense ${ }^{\mathrm{TM}}$ FBG interrogator developed by IFOS. The approach is based on a parallel photonic processing architecture which has the near-term potential to combine high channel counts $(>100$ sensors on a single fiber), high resolution (sub-microstrain), and high speed $(>5 \mathrm{kHz})$ with miniaturized footprint. These features will become increasingly attractive as we seek to increase the sensor number and response speed of our robot system. The ultimate goal is to have the interrogator integrated into the robotic structure as a part of a monitor and control system.

As previously discussed, the application of strain on each FBG produces a shift in the wavelength that is linearly proportional to the strain. An FBG interrogator is used to precisely measure, for each FBG, the reflected wavelength shift and thus the strain applied to that FBG. Interrogators can be tunable (examining each FBG sequentially) or parallel processing in nature - the latter approach, which forms the basis of the IFOS system, has advantages in terms of speed particularly when dealing with many sensors.

The optical interrogator combines (a) optical signal processing (broadband light source, optical circulator, passive photonic parallel processing chip and photo-detector array) with (b) post-detection electronics, and (c) control and monitoring subsystems as shown in Figure 13. Operation is as follows. The broadband source sends light through the optical circulator to an array of FBGs, each of which reflects a different Bragg wavelength. The reflected light is then returned through the optical circulator to the photonic processor which both demultiplexes the light and provides the basis for a ratiometric approach to measuring each of the returned wavelengths through conversion to different signals in various outputs from the multi-channel photodetector array. Electronics and software (or firmware) provide the final conversion of the arrayed signals to wavelength and

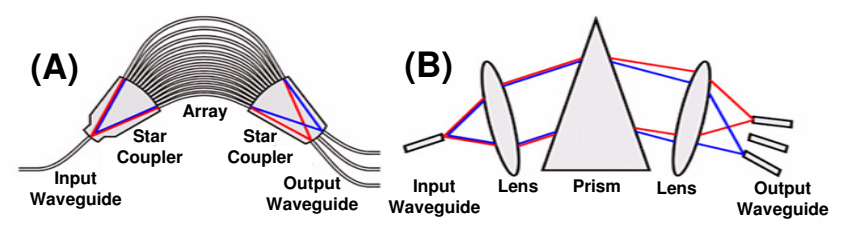

Fig. 14. Schematic representation of the phased-array demultiplexer: (A) Photonic integrated waveguide circuit, (B) Equivalent optical circuit eventually the strain to which each FBG is subjected.

Optical integration is a central technology to achieving substantial cost and size reductions for future integration. The parallel photonic processor is based on Planar Lightwave Circuit (PLC) and phased-array technology (Figure 14) to separate and measure the strain-dependent wavelengths reflected by each of the multiplexed FBGs. IFOS has a pending patent in this area.

\section{CONCLUSIONS AND FUtURE WORK}

This article has described the development of an exoskeletal force sensing robot finger using embedded FBG optical sensors. A rapid prototyping process, shape deposition manufacturing [31], was modified to support the fabrication of hollow, plastic mesh structures with embedded components. The fiber optic sensors were embedded near the base of a cylindrical shell with hexagonal elements for high sensitivity to imposed loads. The resulting structure is light weight and rugged. In initial experiments, the sensorized structure demonstrated measurement of forces of $0.01 \mathrm{~N}$ at frequencies of an order of $20 \mathrm{~Hz}$. With more precise location of the sensors, higher sensitivities should be possible in the future. We also note that any frequency limit is provided by the mechanical finger system, not the interrogator which can measure dynamic strains to $5 \mathrm{kHz}$.

A copper mesh embedded in the structure reduces the amount of viscoelastic creep and provides thermal shielding. A single FBG temperature compensation sensor at the center of the hollow finger helps to reduce the overall sensitivity to thermal variations. However, the central sensor is sufficiently distant from the exterior sensors that changes in temperature produce noticeable transient signals. This effect can be reduced in the future by using a larger number of sensors and locating thermal compensation sensors near the exterior of the structure, where they undergo the same transient thermal strains as the other sensors.

Tests were also conducted to investigate the ability to localize contact forces. Although the ability to localize forces with just four exterior sensors is limited, the results show that the mesh does respond globally to point contacts in a predictable way. With a larger number of sensors, more accurate contact localization will be possible. Increasing the total number of sensors is relatively straightforward as multiple FBG sensors can be located along each fiber with optical multiplexing. 
In parallel to the sensorized finger development, the IFOS team has been developing versions of their interrogator that support a much larger number of sensors and are smaller with higher resolution and long-term stability - in fact future versions are expected to support hundreds of sensors and be sensitive to acoustic and ultrasonic waves. There will remain considerable challenge in processing the data from such systems, but in the long-term such capabilities promise to take robotics far beyond its present sensor-impoverished state.

Furthermore, while the present paper has focused on single-axis FBG strain and temperature sensors in single-core glass fiber sensors, as the technology evolves, we foresee, for example, the potential in robotics for bend sensors based on multi-core fiber supporting FBGs, as well as the use of polymer optical fiber Bragg grating [14] sensors in flexible robotic skins, and eventually a multiplicity of multiplexed physical [7], [9], [10] and chemical [8] fiber-optic sensors.

\section{ACKNOWLEDGMENT}

The authors thank the National Aeronautics and Space Administration (NASA) for financial support for this research through the grant from SBIR contract of NNJ06JA36C to IFOS and subcontract to Stanford's Center for Design Research, and the NASA technical monitor Toby Martin for his support and feedback. Thanks are also especially due to Dr. Behzad Moslehi, CEO and Founder of IFOS for his initiation and support of the project and fruitful discussion. We thank Sanjay Dastoor and Barett Heynman of Stanford University for their suggestions and contributions to this article.

\section{REFERENCES}

[1] F. G. Barth. Spider mechanoreceptors. Current Opinion in Neurobiology 2004, 14:415-422, 2004.

[2] F. G. Barth and J. Stagl. The slit sense organs of arachnids. Zoomorphologie, 86:1-23, 1976.

[3] A. Biccihi and G. Canepa. Optimal design of multivariate sensors. Measurement Science and Technology, 5:319-332, 1994.

[4] A. Biccihi, J. K. Salisbury, and D. L. Brock. Contact sensing from force measurements. International Journal of Robotics Research, 12(3):249-262, 1993.

[5] R. J. Black, K. Chau, G. Chen, B. M. Moslehi, L. Oblea, and K. Sourichanh. Optical fiber gratings for structural health monitoring in high-temperature environments. Proceedings of the SPIE, 6530(62), 2007.

[6] R. J. Black, K. A. Fesler, B. M. Moslehi, B. G. Moslehi, and H. J. Shaw. Distributed grating-assisted bimodal fiber-optic lattice multisensor array networks for smart structures. National Science Foundation Award Number III-9360932, SBIR Final Report, 1994.

[7] R. J. Black, B. M. Moslehi, K. A. Fesler, B. G. Moslehi, and H. J. Shaw. Grating-based fiber-optic sensor arrays for smart aerospace structures. NASA Langley Research Center SBIR Final Report - Phase I Contract NAS 1-20356, 1995.

[8] R. J. Black, B. M. Moslehi, M. Shahriari, and H. J. Shaw. Insitu biosafe fiber-optic carbon dioxide sensors for real-time bioreactor monitoring. NASA Johnson Space Center, SBIR Final Report, 1997.

[9] R. J. Black, B. M. Moslehi, and H. J. Shaw. Temperature compensated magnetic field sensors for optimized materials processing. NASA Marshall Space Flight Center, SBIR Final Report, 1997.

[10] R. J. Black, B. M. Moslehi, K. Toyama, and H. J. Shaw. Gratingbased fiber-optic sensor arrays for smart aerospace structures. NASA Langley Research Center SBIR Final Report - Phase II, 1998.
[11] R. Blickhan and F. G. Barth. Strains in the exoskeleton of spiders. Journals of Comparative Physiology A, 157:115-147, 1985.

[12] K. Chau, B. Moslehi, G. Song, and V. Sethi. Experimental demonstration of fiber bragg grating strain sensors for structural vibration control. Proceedings of the SPIE, Smart Structures and Materials 2004: Sensors and Smart Structures Technologies for Civil, Mechanical, and Aerospace Systems, 5391:753-764, 2004.

[13] K. Chau, P. Qiao, W. Lestari, R. J. Black, and B. M. Moslehi. Highspeed, high-resolution fiber bragg grating matrix structural health monitoring systems. Proceedings of the SPIE, 6530(63), 2007.

[14] H. Dobb, D. J. Webb, K. Kalli, A. Argyros, M. C. J. Large, and M. A. van Eijkelenborg. Continuous wave ultraviolet light-induced fibre bragg gratings in few-and single-moded microstructured polymer optical fibres. Optics Letters, 30(24):3296-3298, 2006.

[15] A. Dollar, C. R. Wagner, and R. D. Howe. Embedded sensors for biomimetic robotics via shape deposition manufacturing. Proceedings of the first IEEE/RAS-EMBS International Conference on Biomedical Robotics and Biomechatronics (BioRob2006), 2006.

[16] W. Griffin, W. M. Provancher, and M. R. Cutkosky. Feedback strategies for telemanipulation with shared control of object handling forces. Presence, MIT Press, 14(6):720-731, 2005.

[17] T. C. Hales. The honeycomb conjecture. 1999, Available: http://arxiv.org/abs/math.MG/9906042, 1999.

[18] X. C. Li and F. Prinz. Metal embedded fiber bragg grating sensors in layered manufacturing. Journal of Manufacturing Science and Engineering, 125:577-585, 2003.

[19] S. Magne, S. Rougeault, M. Vilela, and P. Ferdinand. State-ofstrain evaluation with fiber bragg grating rosettes: application to discrimination between strain and temperature effects in fiber sensors. Applied Optics, 36(36):9437-9447, 1997.

[20] D. T. Moran, K. M. Chapman, and R. S. Ellis. The fine structure of cockroach campaniform sensilla. The Journal of Cell Biology, 48:155173, 1971.

[21] W. W. Morey, G. Meltz, and J. M.. Weiss. Recent advances in fiber bragg grating instrumentation systems. SPIE, Self Calibrated Intelligent Optical Sensors and Systems, 2594:90-98, 1995.

[22] B. M. Moslehi, R. J. Black, K. Toyama, and H. J. Shaw. Multiplexible fiber-optic strain sensor system with temperature compensation capability. Divisions 1-3 U.S. Patents 6,597,822, issued July 22, 2003, 6,788,835, issued Sept 7, 2004, and 6,895,132, issued May 17, 2005.

[23] S. Pal, J. Mandal, T. Sun, K. T. V. Grattan, M. Fokine, F. Carlsson, P. Y. Fonjallaz, S. A. Wade, and S. F. Collins. Characteristics of potential fibre bragg grating sensor-based devices at elevated temperatures. Measurement Science and Technology, (14):1131-1136, 2003.

[24] I. Peterson. The honeycomb conjecture. Science News, 156(4):60, 1999.

[25] Y. J. Rao. In-fibre bragg grating sensors. Measurement Science and Technology, 8(355-375), 1997.

[26] E-A. Seyfarth, W. Eckweiler, and K. Hammer. Proprioceptors and sensory nerves in the legs of a spider, cupiennius salei (arachnida, araneida). Zoomorphologie, 105:190-196, 1985.

[27] D. S. Smith. The fine structure of haltere sensilla in the blowflycalliphora erythrocephala (meig.) with scanning electron microscopic observations on the haltere surface. Tissue and Cell, 1:443-484, 1969.

[28] J. S. Son, M. R. Cutkosky, and R. D. Howe. Comparison of contact sensor localization abilities during manipulation. Proceedings of 1995 IEEE/RSJ International Conference on Intelligent Robots and Systems, 2:96-103, 1995.

[29] E. Udd. Fiber optic smart structure. Proceedings of the IEEE, 84(6), 1996.

[30] R. M. Voyles, G. Fedder, and P. K. Khosla. Design of a modular tactile sensor and actuatator based on an electrorheological gel. Proceedings of the 1996 IEEE International Conference on Robotics and Automation, 1:13-17, 1996.

[31] L. E. Weiss, R. Merz, F. B. Prinz, G. Neplotnik, P. Padmanabhan, L. Schultz, and K. Ramaswami. Shape deposition manufacturing of heterogenous structures. Journal of Manufacturing Systems, 16(4):239-248, 1997.

[32] M. G. Xu, J-L. Archambault, L. Reekie, and J. P. Dakin. Discrimination between strain and temperature effects using dual-wavelength fibre grating sensors. Electronics Letters, 30(13):1085-1087, 1994.

[33] M.G. Xu, L. Dong, L. Reekie, J.A. Tucknott, and J.L. Cruz. Temperature-independent strain sensor using a chirped bragg gratingin a tapered optical fibre. Electronics Letters, 31(10):823-825, 1995. 